\title{
Structure, ferroelectric and local piezoelectric properties of KNN- based perovskite ceramics
}

\author{
E.D. Politova $^{1}$, G.M. Kaleva ${ }^{1}$, A.V. Mosunov ${ }^{1}$, N.V. Sadovskaya ${ }^{1}$, D.A. Kiselev ${ }^{2}$,
} A.M. Kislyuk ${ }^{2}$, S.Yu. Stefanovich ${ }^{1,3}$, P.K. Panda ${ }^{4}$

\author{
${ }^{1}$ L.Ya.Karpov Institute of Physical Chemistry, Moscow 105064 Russia, \\ politova@nifhi.ru \\ ${ }^{2}$ National University of Science and Technology "MISiS”, Moscow 119991 Russia, \\ ${ }^{3}$ Lomonosov Moscow State University, Moscow 119992 Russia, \\ ${ }^{4}$ National Aerospace Laboratories, Kodihalli, Bangalore-560017 India
}

Lead-free ferroelectric materials continue to be intensively studied in order to replace widely used lead-based ones [1-4]. We studied influence of cation substitutions and preparation conditions on structure, microstructure, ferroelectric, and local piezoelectric properties of solid solutions in the systems on the base of sodium-potassium niobate $\left(\mathrm{K}_{0.5} \mathrm{Na}_{0.5}\right) \mathrm{NbO}_{3}(\mathrm{KNN})$ with perovskite structure [5-7].

Ceramic samples in the $\mathrm{KNN}$-based systems were prepared by the two-step solid-state reaction method at temperatures of 900-1500 K. Solid solutions with $\mathrm{Ba}^{2+}, \mathrm{Li}^{+}, \mathrm{Ca}^{+}$in A- and with $\mathrm{Cu}^{2+}, \mathrm{Mn}^{3+}, \mathrm{Ni}^{2+}, \mathrm{Fe}^{3+}$ cations in B-sites of perovskite lattice were prepared. To improve density of ceramics overstoichiometric additives $(\mathrm{KCl}, \mathrm{LiF}$ and $\mathrm{ZnO})$ were used.

To characterize the samples, complex of physico-chemical methods was used: the X-ray Diffraction, Scanning Electron Microscopy (SEM), Second Harmonic Generation (SHG), Dielectric Spectroscopy (DS), and Atomic Force Microscopy in Piezoresponse Force Mode (PFM) methods.

The observed unit cell volume changes in modified KNN-based ceramics depended on ionic radii of substituting cations. Mean size of grains changed from $\sim 3$ till $\sim 20 \mu \mathrm{m}$ in compositions containing low melting additives $\mathrm{KCl}$ and $\mathrm{LiF}$. Ferroelectric phase transitions at $\sim 400$ and near $700 \mathrm{~K}$ were confirmed using the DS and SHG methods. At temperatures higher than $\sim 900 \mathrm{~K}$ effects of dielectric relaxation caused by formation of oxygen vacancies were observed in ceramics with aliovalent substitutions.

Increase in the spontaneous polarization value while non monotonous changes in dielectric permittivity values and were revealed for modified ceramics at the room temperature. Ferroelectric polarization switching at nanoscale was observed using PFM method, and in KNN-based ceramics high values of effective $d_{33}$ piezoelectric coefficient $200-300 \mathrm{pm} / \mathrm{V}$ reached. The results obtained confirm the statement of prospects of creating new lead-free materials on the base of modified KNN- based compositions.

Acknowledgment. The work was supported by the Russian Foundation for Basic Research (Projects 16-53-48009, 18-03-00372).

1. I. Coondoo, N. Panwar, A. Kholkin, J. Adv. Dielectr. 3, 1330002 (2013).

2. P.K. Panda and B. Sahoo, Ferroelectrics 474, 128 (2015).

3. D. Alikin, A. Turygin, A. Kholkin and V. Shur, Materials 10, 47 (2017).

4. J. Rödel and J. Li, MRS Bulletin 43, 576 (2018).

5. E.D. Politova, G.M. Kaleva, N.V. Golubko, et al., Ferroelectrics 489, 147 (2015).

6. E.D. Politova, E.A. Fortalnova, M.G. Safronenko, et al., Ferroelectrics 518, 109 (2017).

7. E.D. Politova, N.V. Golubko, G.M. Kaleva, et al., J. Adv. Diel. 8, 1850004 (2018). 